\title{
Mechanic's hands revisited: is this sign still useful for diagnosis in patients with lung involvement of collagen vascular diseases?
}

Erei Sohara', Takeshi Saraya', Shinji Sato², Naoki Tsujimoto', Takayasu Watanabe', Saori Takata', Yasutaka Tanaka', Haruyuki Ishii ${ }^{1}$, Hajime Takizawa ${ }^{1}$ and Hajime Goto ${ }^{1 *}$

\begin{abstract}
Background: The presence of "mechanic's hands" is one of the clinical clues for collagen vascular diseases. However, the exact relevance of "mechanic's hands" in collagen vascular diseases has not been well documented. The aim of this study was to clarify the relevance of "mechanic's hands" to collagen vascular diseases including various skin lesions and interstitial pneumonia.

Methods: A retrospective review of the medical records of patients with "mechanic's hands" at our hospital between April 2011 and December 2012 was conducted. A PubMed search was also conducted using the term "mechanic's hands".

Results: Four patients in our institution and 40 patients obtained from PubMed who had "mechanic's hands" were identified. The most frequent diseases were DM/amyopathic DM ( $n=24,54.5 \%)$ and anti-ARS syndrome $(\mathrm{n}=17,38.6 \%)$. In these patients, the major skin lesions associated with "mechanic's hands" were periungual erythema ( $n=23,52.3 \%)$, Gottron's sign $(n=17,38.6 \%)$, heliotrope rash $(n=10,22.7 \%)$, Raynaud's phenomenon $(n=9,20.5 \%)$, and anti-ARS syndrome $(n=17,38.6 \%)$. Six cases (2 DM, 4 anti-ARS syndrome) had only "mechanic's hands". Antibodies to anti-ARS ( $n=24)$ were Jo-1 $(n=19)$, PL-7 $(n=3)$, OJ $(n=1)$, and PL-12 $(n=1)$.

Conclusion: The presence of "mechanic's hands" together with diverse skin lesions could be a clinical clue to the diagnosis of lung involvement associated with collagen vascular diseases, especially in anti-ARS syndrome or DM/amyopathic DM.
\end{abstract}

Keywords: Anti-aminoacyl-transfer RNA synthetase (ARS) syndrome, Dermatomyositis, Mechanic's hands, Skin lesions

\section{Background}

In 1979, Stahl et al. [1] described "mechanic's hands" as a hyperkeratotic eruption on the ulnar aspect of the thumb and radial aspect of the index finger, with desquamation and rhagades. The presence of "mechanic's hands" has been reported to be highly relevant in patients with collagen vascular-related interstitial pneumonia, dermatomyositis, systemic lupus erythematosus, and mixed connective tissue disease. In the modern era, anti-ARS syndrome has emerged as a new clinical entity

\footnotetext{
* Correspondence: h510@ks.kyorin-u.ac.jp

${ }^{1}$ Department of Respiratory Medicine, Kyorin University School of Medicine, 6-20-2 Shinkawa, Mitaka City, Tokyo 181-8611, Japan

Full list of author information is available at the end of the article
}

associated with interstitial pneumonia. However, the exact relevance of "mechanic's hands" in these various diseases has not been well reported. Four patients with amyopathic dermatomyositis or dermatomyositis with "mechanic's hands" are reported, and 40 previously reported cases are reviewed.

\section{Methods}

Patients who presented to the Kyorin University School of Medicine (Mitaka City, Tokyo, Japan) who were consecutively admitted to the Department of Respiratory Medicine with "mechanic's hands" based on the criteria of Stahl et al. were investigated [1]. A retrospective study over a 20-month span from April 2011 was conducted.

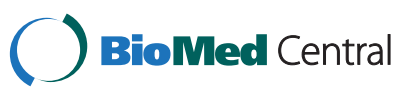


Patients who satisfied the criteria for inflammatory myositis, polymyositis, and dermatomyositis proposed by Bohan and Peter's [2] and Tanimoto et al. [3], respectively, were enrolled. The medical literature was also searched using PubMed to identify reports of "mechanic's hands". This retrospective study was approved by the Ethics Board of Kyorin University.

\section{Results}

In our institution, 10 consecutive patients with DM $(n=9)$ or DM/SLE overlap syndrome $(n=1)$ were identified, of whom four had "mechanic's hands" (Figure 1 of case 2).

Case 1 was a 51-year-old man who presented to our emergency department with a 10-day history of dyspnea and dry cough. He had a medical history of atopic dermatitis and bronchial asthma. His vital signs were as follows: blood pressure $130 / 80 \mathrm{mmHg}$, pulse rate 96 beats/min, temperature $36.8^{\circ} \mathrm{C}$, respiratory rate 24 breaths $/ \mathrm{min}$, and oxygen saturation $99 \%$ at $6 \mathrm{~L} / \mathrm{min}$ oxygen delivered via a mask. Physical examination was normal except for inspiratory fine crackles in bilateral lower lung fields posteriorly. He had skin lesions of "mechanic's hands" on the ulnar aspect of the thumb and radial aspect of the index finger, as well as erythema at the nose, anterior portion of the neck or back, and olecranon 1 month prior to coming to our hospital. The skin over the palmar and dorsal aspects of the finger joints of both hands showed red-purple, keratotic, atrophic erythema, suggesting Gottron's sign, together with periungual erythema. Dark red erythema with hyperkeratosis was found on the anterior portion of the neck and back, compatible with the V-neck sign and shawl sign, respectively.

Laboratory data revealed slightly elevated levels of Creactive protein $(\mathrm{CRP})(2.8 \mathrm{mg} / \mathrm{dL})$ and aldolase $(22.6 \mathrm{U} / \mathrm{L})$. Moderate elevations of serum lactate dehydrogenase (LDH

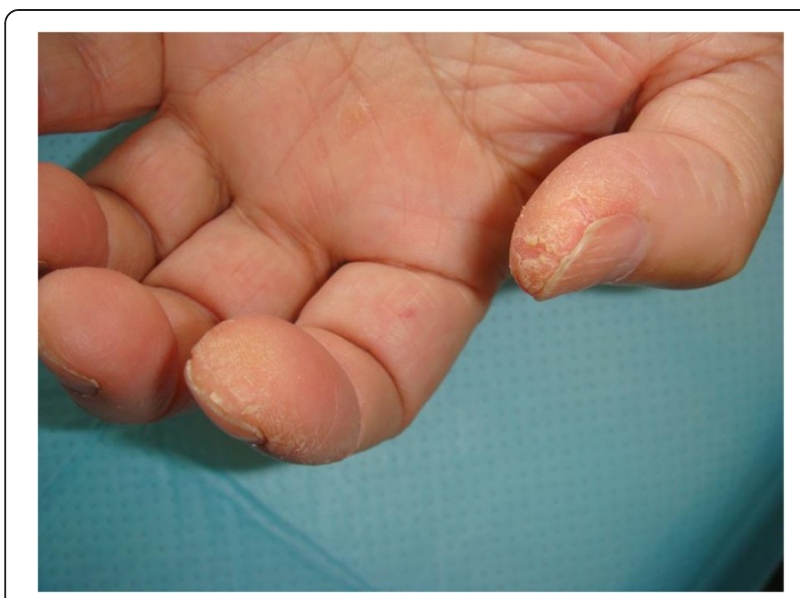

Figure 1 Patient 3 has fissures and roughness with hyperkeratosis and scaling on the pulp of the thumb and the radial aspect of the index finger.
(413 IU/L), KL-6 (883 U/mL), and SP-D (222 ng/mL) were also noted. Arterial blood gas analysis with oxygen at $6 \mathrm{~L} / \mathrm{min}$ via mask showed hypoxemia (80.9 Torr), but other data were normal $\left(\mathrm{pH} 7.444, \mathrm{pCO}_{2} 37.8\right.$ Torr, and $\left.\mathrm{HCO}_{3}-24.1 \mathrm{mEq} / \mathrm{L}\right)$. Antibody to clinical amyopathic dermatomyositis (CADM)-140 autoantigen antibody (titer 1:232) was detected, but no anti-aminoacyl-transfer RNA synthetase (anti-ARS) antibodies including Jo-1 were detected. Thoracic CT showed an organizing pneumonia (OP) pattern.

He satisfied five of the nine Japan College of Rheumatology criteria [3], and was thus diagnosed with amyopathic dermatomyositis (DM) with interstitial pneumonia (IP). He was immediately treated with intravenous steroid pulse therapy (methylprednisolone $1 \mathrm{~g}$ /day for 3 days), followed by oral maintenance steroid therapy with a dose of $0.8 \mathrm{mg} / \mathrm{kg} /$ day combined with oral cyclosporine $(4 \mathrm{mg} / \mathrm{kg} /$ day). A few days after initiation of treatment, his skin lesions and radiological abnormalities disappeared almost completely, while his respiratory failure improved gradually within a month, and he was discharged uneventfully.

Case 2 was a 71-year-old male artist (painter) who presented to our hospital due to exertional dyspnea and low-grade fever for a month. His medical history included a thyroid tumor and prostate cancer at 26 and 62 years old, respectively. An abnormality was noted on a chest radiograph 10 months prior to coming to our hospital, but he denied any diagnostic procedures. His vital signs were as follows: blood pressure $120 / 78 \mathrm{mmHg}$, pulse rate 60 beats/ min, temperature $36.6^{\circ} \mathrm{C}$, respiratory rate 20 breaths $/ \mathrm{min}$, and oxygen saturation $94 \%$ on ambient air. Physical examination was normal except for inspiratory fine crackles in posterior lower lung fields bilaterally. He had "mechanic's hands", Gottron's sign, and periungual erythema.

Laboratory examination showed mild elevation of LDH (325 UI/L) and moderate elevation of SP-D (223 ng/mL). KL-6 was markedly elevated (1,817 U/mL). Anti-Jo-1 antibody was detected (titer 1:174.9), but no other autoantibodies, including anti-ARS antibodies, were detected. Thoracic CT showed curvilinear opacities in the right lower lobe, suggesting non-specific interstitial pneumonia (NSIP) pattern. He was clinically diagnosed with amyopathic DM with IP and was treated with oral prednisolone $0.8 \mathrm{mg} / \mathrm{kg} /$ day. His skin lesions and radiological abnormalities improved gradually, and he was successfully discharged on hospital day 30 .

Case 3 was a 31-year-old man who presented to our hospital with a 2- month history of hyperkeratotic papules on bilateral fingers and erythema on the nose. He had a low-grade fever, arthralgia, myalgia, and muscle weakness in the upper extremity for 1 week. His vital signs were normal except for low-grade fever $\left(37.2^{\circ} \mathrm{C}\right)$. He had "mechanic's hands" on the ulnar aspect of the thumb and 
radial aspect of the index finger and Gottron's sign or periungual erythema of bilateral fingers.

Laboratory examinations were normal except for slightly elevated levels of LDH (316 IU/L), while the presence of antibody to CADM-140 autoantigen (titer $1 ; 231$ ) was noted, but no other auto-antibodies were detected. Thoracic CT showed patchy consolidation with bronchiectasis at the bottom of the lung, predominantly in the right thorax, suggesting NSIP pattern. He satisfied five of the nine Japan College of Rheumatology criteria [3], and biopsy of the erythema of his left index finger showed superficial perivascular and interface dermatitis, which was histologically identical to that of DM. He was thus diagnosed with amyopathic DM with IP, and discharged on hospital day 3 without treatment. He was in good health for the next 5 months without treatment, and

Table 1 Clinical features of patients with mechanic's hands reported previously and the present four patients

\begin{tabular}{|c|c|c|c|c|c|c|c|c|c|c|c|c|c|}
\hline Year & Age (y) & Sex & $\begin{array}{l}\text { Gottron's } \\
\text { sign }\end{array}$ & $\begin{array}{l}\text { Heliotrope } \\
\text { rash }\end{array}$ & $\begin{array}{l}\text { Shawl } \\
\text { sign }\end{array}$ & $\begin{array}{l}\text { V-neck } \\
\text { sign }\end{array}$ & Raynaud's & $\begin{array}{l}\text { Periungual } \\
\text { erythema }\end{array}$ & $\begin{array}{l}\text { Myalgia or } \\
\text { weakness }\end{array}$ & Auto-antibody & Diagnosis & IP & Ref \\
\hline 1994 & 69 & $\mathrm{~F}$ & - & - & - & - & - & - & + & Jo-1 & $\mathrm{DM}$ & + & {$[2]$} \\
\hline 1996 & 7 & M & + & - & - & - & + & + & + & ANA, ds-DNA, RF & SDM & N.A & {$[3]$} \\
\hline 2000 & 67 & M & + & + & + & + & - & + & - & - & $\mathrm{DM}$ & + & {$[4]$} \\
\hline 2001 & 42 & M & + & - & - & - & - & - & + & ANA & $\mathrm{DM}$ & - & {$[5]$} \\
\hline 2002 & 41 & M & - & + & - & - & + & - & + & Jo-1 & Anti-ARS synd & + & {$[6]$} \\
\hline 2002 & 40 & $\mathrm{~F}$ & - & + & - & - & - & - & + & Jo-1 & Anti-ARS synd & + & {$[6]$} \\
\hline 2002 & 30 & M & - & + & - & - & - & - & - & Jo-1 & Anti-ARS synd & + & {$[6]$} \\
\hline 2003 & 48 & $\mathrm{~F}$ & - & - & - & - & - & + & - & RNP & RA & + & {$[7]$} \\
\hline 2003 & 55 & M & + & + & - & - & - & - & - & Jo-1, RNP & $\mathrm{DM}$ & + & {$[7]$} \\
\hline 2003 & 29 & $\mathrm{~F}$ & - & - & - & - & - & - & - & Jo-1 & $\mathrm{DM}$ & + & {$[7]$} \\
\hline 2004 & 56 & $\mathrm{~F}$ & + & - & - & - & - & - & - & PM-SCL & SDM & + & {$[8]$} \\
\hline 2005 & 62 & $\mathrm{~F}$ & + & - & - & - & - & - & - & PL-7 & $\mathrm{DM}$ & + & {$[9]$} \\
\hline 2005 & 59 & $\mathrm{~F}$ & - & - & - & - & + & - & + & Jo-1 & Anti-ARS synd & + & [10] \\
\hline 2006 & 30 & M & + & + & - & - & + & - & - & PL-7 & Anti-ARS synd & + & [11] \\
\hline 2007 & 41 & M & + & - & - & + & - & - & - & Jo-1 & Anti-ARS synd & + & [12] \\
\hline 2007 & 37 & $\mathrm{~F}$ & - & - & - & - & + & - & + & Jo-1 & Anti-ARS synd & + & [13] \\
\hline 2007 & 73 & $\mathrm{~F}$ & - & - & - & - & + & - & - & Jo-1 & Anti-ARS synd & + & [13] \\
\hline 2007 & 49 & $\mathrm{~F}$ & - & - & - & + & - & - & - & Jo-1 & Anti-ARS synd & + & [13] \\
\hline 2008 & 56 & $\mathrm{~F}$ & - & - & - & - & - & - & - & ANA, Jo-1, SS-A & Anti-ARS synd & + & [14] \\
\hline 2009 & 56 & M & + & - & - & - & - & + & - & PL-7 & DM & + & [15] \\
\hline 2009 & 64 & $\mathrm{~F}$ & + & - & + & - & - & + & + & - & $\mathrm{DM}$ & + & [15] \\
\hline 2009 & 44 & $\mathrm{~F}$ & + & - & - & - & - & + & - & ANA & $\mathrm{DM}$ & - & [15] \\
\hline 2009 & 56 & M & - & + & - & - & - & + & + & ANA & $\mathrm{DM}$ & + & [15] \\
\hline 2009 & 65 & $\mathrm{~F}$ & - & + & - & - & - & + & + & ANA & $\mathrm{DM}$ & + & [15] \\
\hline 2010 & 54 & $\mathrm{~F}$ & - & - & - & - & - & - & - & OJ & Anti-ARS synd & + & [16] \\
\hline 2010 & 67 & $\mathrm{~F}$ & - & - & - & - & - & + & - & SS-A, Jo-1 & Anti-ARS synd & + & [17] \\
\hline 2010 & 48 & $\mathrm{~F}$ & + & - & - & - & + & + & - & SS-A, Jo-1, ASMA & Anti-ARS synd & + & [18] \\
\hline 2011 & 64 & $\mathrm{~F}$ & - & - & - & - & + & - & + & Jo-1 & Anti-ARS synd & + & [19] \\
\hline 2011 & 43 & M & - & - & - & - & - & - & + & Jo-1 & Anti-ARS synd & + & [20] \\
\hline 2012 & 21 & M & - & - & - & - & + & - & + & PL-12 & Anti-ARS synd & + & [21] \\
\hline 2012 & 79 & M & - & - & - & - & - & - & + & Jo-1 & Anti-ARS synd & + & [22] \\
\hline Case 1 & 51 & M & + & - & + & + & - & + & + & CADM-140 & ADM & + & \\
\hline Case 2 & 71 & M & + & - & - & - & - & + & - & Jo-1 & ADM & + & \\
\hline Case 3 & 31 & M & + & - & - & - & - & + & + & CADM-140, AQP-4 & $\mathrm{DM}$ & + & \\
\hline Case 4 & 77 & M & + & + & + & + & - & + & + & ANA & $\mathrm{DM}$ & - & \\
\hline
\end{tabular}

ANA: anti nuclear antibody, AQP-4: aquaporin 4, ARS: aminoacyl-transfer RNA synthetase, ASMA: anti-smooth muscle antibody, CADM: clinical amyopathic dermatomyositis, DM: dermatomyositis, ds-DNA: double strand-DNA, IP: interstitial pneumonia, NA: not available, NSIP: non specific interstitial pneumonia, OP: organizing pneumonia, PM: polymyositis, RA: rheumatoid arthritis, RF: rheumatoid factor, RNP: ribonucleoprotein, SDM: sclerodermatomyositis, UIP: usual interstitial pneumonia. 
most skin lesions and abnormal findings on thoracic CT disappeared spontaneously in this period, but 6 months after diagnosis, he returned to our hospital with abrupt onset of photophobia, dysphagia, aphasia, and numbness of the left sided-trunk, suggesting a central nervous system disorder. T2-weighted magnetic resonance imaging (MRI) demonstrated hyperintense lesions at the periventricular white matter that extended longitudinally in the spinal cord from C5 to Th8 (figure not shown). The cerebrospinal fluid showed pleocytosis (total cell count 2 cells/ $\mu \mathrm{L}$, total protein $46.9 \mathrm{mg} / \mathrm{dL}$ ), and serum anti-aquaporin-4 (AQP-4) antibody was positive. He was finally diagnosed with amyopathic DM accompanied by neuromyelitis optica (NMO). He was then treated intensively with four cycles of steroid pulse therapy (prednisolone $1 \mathrm{~g} /$ day for 3 days), followed by maintenance therapy with oral prednisolone $(0.5 \mathrm{mg} / \mathrm{kg} /$ day $)$, and discharged uneventfully.

Case 4 was a 77-year-old man who presented to his local hospital due to erythema of the face and body. The erythema progressed, and muscle weakness in the thigh, as well as eyelid edema, emerged. Two months after his first visit to his local hospital, he came to our hospital. His vital and physical examinations were normal except for cutaneous lesions such as "mechanic's hands", Gottron's sign, periungual erythema, heliotrope rash, shawl sign, and V-neck sign. Laboratory examinations showed slightly elevated white blood cells (WBCs) $(9100 / \mu \mathrm{L})$ and LDH (311 IU/L), and marked elevation of CK (509 IU/L). Antinuclear antibody was detected (titer 1:640), but no other auto-antibodies were detected. He satisfied four of the nine Japan College of Rheumatology criteria [3]. He had no lung involvement and was thus diagnosed with DM. At the same time, he was diagnosed with gastric cancer, which was treated by total gastrectomy. After surgery, he suffered from exacerbation of myositis, but intravenous steroid pulse therapy (prednisolone $1 \mathrm{~g}$ /day for 3 days), followed by intravenous maintenance therapy with prednisolone $(1 \mathrm{mg} / \mathrm{kg} /$ day $)$, led to complete resolution of the myositis, and he was transferred to another hospital.

Along with an intensive review of the previous literature, 44 patients with "mechanic's hands", including the four present cases, were identified. Their age was $51.5 \pm$ 16.3 (mean \pm S.D) years and ranged from 7 to 79 years,

Table 2 Diseases related to mechanic's hands

\begin{tabular}{lc}
\hline Disease & $\mathbf{n}(\%)$ \\
\hline DM/amyopathic DM & $24(54.5)$ \\
Anti-ARS syndrome & $17(38.6)$ \\
SDM & $2(4.5)$ \\
RA & $1(2.3)$ \\
\hline
\end{tabular}

ARS: aminoacyl-transfer RNA synthetase, DM: dermatomyositis, SDM: sclerodermatomyositis, PM: polymyositis, RA: rheumatoid arthritis.
Table 3 Skin lesions with mechanic's hands

\begin{tabular}{lcc}
\hline Skin lesion & $\mathbf{n}$ & $\mathbf{( \% )}$ \\
\hline Periungual erythema & 23 & $(52.3)$ \\
Gottron's sign & 17 & $(38.6)$ \\
Heliotrope rash & 10 & $(22.7)$ \\
V-neck sign & 5 & $(11.4)$ \\
Shawl sign & 4 & $(9.1)$ \\
None & 6 & $(13.7)$ \\
\hline
\end{tabular}

with a male to female ratio of 18:26 (Table 1) [4-25]. They had diverse diseases, such as DM/amyopathic DM $(\mathrm{n}=24,54.5 \%)$, anti-ARS syndrome $(\mathrm{n}=17,38.6 \%)$, sclerodermatomyositis $(\mathrm{n}=2,4.5 \%)$, and rheumatoid arthritis $(\mathrm{n}=1,2.3 \%)$ (Table 2). Skin lesions other than "mechanic's hands" were noted, such as periungual erythema ( $\mathrm{n}=23,52.3 \%)$, Gottron's sign $(\mathrm{n}=17,38.6 \%)$, heliotrope rash $(n=10,22.7 \%)$, the V-neck sign $(n=5$, $11.4 \%)$, and the shawl sign ( $\mathrm{n}=4,9.1 \%)$ (Table 3). Importantly, six patients (two DM, four anti-ARS syndrome) had "mechanic's hands" as the sole skin lesion. The other 38 patients had multiple skin lesions with the following number of lesions other than "mechanic's hands": one (20 patients), two (11 patients), three (four patients), four (one patient), and five (two patients). The combinations of skin lesions are summarized in Table 4. The most common combination pattern of multiple skin lesions was periungual erythema and Raynaud's phenomenon. On the other hand, two or more skin lesions with "mechanic's hands" tend to be recognized as a combination of Gottron's sign/periungual erythema and heliotrope rash/periungual erythema, in this order (Table 4).

Table 4 Combinations of skin lesions excluding mechanic's hands

\begin{tabular}{ll}
\hline One & $\mathbf{n}$ \\
\hline Periungual erythema & 9 \\
Raynaud's phenomenon & 5 \\
Gottron's sign & 3 \\
Heliotrope rash & 2 \\
V-neck sign & 1 \\
Two & 5 \\
\hline Gottron's sign/Periungual erythema & 3 \\
Heliotrope rash/Periungual erythema & 1 \\
Gottron's sign/Heliotrope rash & 1 \\
Gottron's sign/V-neck sign & 1 \\
Heliotrope rash/Raynaud's phenomenon & 2 \\
Three & 1 \\
\hline Gottron's sign/Raynaud's phenomenon/Periungual erythema & 1 \\
Gottron's sign/Raynaud's phenomenon/Heliotrope rash &
\end{tabular}


Interestingly, the proportion of patients with IP who had one $(n=17)$, two $(n=10)$, and three $(n=4)$ skin lesions in addition to "mechanic's hands" was high, $85 \%, 91 \%$, and $100 \%$, respectively. This fact suggested that the presence of diverse skin lesions together with "mechanic's hands" raises the possibility of lung involvement associated with collagen vascular diseases.

As for the auto-antibodies, 24 had anti-ARS $(n=24)$, which consisted of Jo-1 $(n=19)$, PL-7 $(n=3)$, OJ $(n=1)$, or PL-12 $(\mathrm{n}=1)$, followed by anti-nuclear antibody $(\mathrm{n}=14)$, SS-A $(\mathrm{n}=3)$, and anti-RNP antibody $(\mathrm{n}=2)$. Interstitial pneumonia and myositis were common, seen in 38 (86.4\%) and 24 patients (54.5\%), respectively. AntiARS syndrome (all 17 patients) and DM (24 of 26 patients, including amyopathic DM or SDM) patients had a higher morbidity rate from interstitial pneumonia $(100 \%$ and $79.2 \%$, respectively).

\section{Discussion}

In the past two decades, the anti-ARS syndrome has been defined as the presence of one of the eight antisynthetase autoantibodies (anti-Jo-1, anti-PL-7, anti-PL-12, anti-EJ, anti-OJ, anti-KS, anti-Zo, anti-Ha) [23,26], but, except for Jo-1 antibody, they are detectable only in special laboratories. Anti-ARS syndrome has at least one of the following three clinical features: interstitial lung disease, inflammatory myopathy, or inflammatory polyarthritis [23]. However, previous reports noted that anti-ARS syndrome does not always show evidence of myositis, and a small percentage (2-11\%) of patients with dermatomyositis does not have muscle involvement (so-called amyopathic dermatomyositis or dermatomyositis sine myositis) [14]. Thus, more attention should be paid to skin lesions in the diagnostic process. In our institution, there were no patients with anti-ARS syndrome during the study period.

"Mechanic's hands" were first described by Stahl et al. in 1979 [1], with similar findings to manual workers, clinically resembling hand eczema [19]; however, the following differential points were noted: 1) there was no evidence of pruritus and vesicles; 2) the lesions were usually symmetrical, not related to the dominant hand; 3) involved the ulnar aspect of the thumb and the radial aspect of the fingers, most prominently on the index and middle fingers; and 4) liquefaction degeneration and colloid bodies were typical pathological features of "mechanic's hands". Previous reports showed that only $33 \%$ of patients with DM [1,27], as well as $30 \%$ to $70 \%$ of patients with anti-ARS syndrome [8], had "mechanic's hands". In this regard, in our institution, $40 \%$ of patients with DM had "mechanic's hands", a prevalence similar to that of previous reports. The present study demonstrated that the majority of patients with "mechanic's hands" had anti-ARS syndrome or DM/amyopathic
DM. However, this study had some limitations because it was a retrospective study, and one case series described by Sato et al. [25] was included in the present study that had a large number of patients $(n=9)$ with DM having "mechanic's hands". However, since the advent of the new clinical entity of anti-ARS syndrome in the last two decades, no study has been published describing the clinical significance of "mechanic's hands". In this regard, the present study showed that anti-ARS syndrome should be considered a leading cause of "mechanic's hands". Of note, six cases of patients with DM or amyopathic DM had "mechanic's hands" as the sole skin lesion. Furthermore, only half of the patients in the present study showed myositis. Taken together, these facts suggest that the presence of "mechanic's hands" serves as a high-yield guide in the diagnosis for DM/amyopathic DM or antiARS syndrome, which might be useful in cases that lack clinically apparent myositis and/or in institutions where detection of antisynthetase autoantibodies other than Jo-1 antibody is not available.

Although our study had some limitations in that all our patients had been admitted to the Department of Respiratory Medicine, lung involvement in dermatomyositis and idiopathic inflammatory myopathy occurs in 5-30\% [10], while it is more common in anti-ARS syndrome $(80 \%$ $100 \%$ ) $[24,27]$, as in the present study. Although no report has been published about "mechanic's hands" associated with idiopathic interstitial pneumonia, Watanabe et al. demonstrated that antisynthetase antibody-positive cases accounted for $6.6 \%$ of idiopathic interstitial pneumonia cases [28]. From this perspective, when we encounter patients with IP, recognition of common combinations of diverse skin lesions, as well as "mechanic's hands", might be a clinical clue to the diagnosis of collagen vascular diseases associated with lung involvement.

\section{Conclusion}

The presence of "mechanic's hands" together with diverse skin lesions could be a clinical clue to the diagnosis of lung involvement with collagen vascular diseases, especially in anti-ARS syndrome or DM/amyopathic DM.

\section{Abbreviations}

ARS: Anti-aminoacyl-transfer RNA synthetase syndrome.

\section{Competing interests}

The authors declare that they have no competing interests.

\section{Authors' contributions}

ES and TS mainly wrote the manuscript. NT, TW, ST, YT, HI, HT, and HG managed the patients. SS examined the titer of anti-synthetase antibodies. All authors read and approved the final manuscript.

\section{Acknowledgements}

Written consent was obtained from the patients or their relative for publication of this study. 


\section{Disclosures}

The authors have nothing to report regarding "Disclosures of financial interest" and declare that we are not thinking of redundant or duplicate publication. The authors declare that all authors have agreed to the conditions noted on the manuscript.

\section{Author details}

'Department of Respiratory Medicine, Kyorin University School of Medicine, 6-20-2 Shinkawa, Mitaka City, Tokyo 181-8611, Japan. ${ }^{2}$ Division of Rheumatology, Department of Internal Medicine, Tokai University School of Medicine, Tokai University School of Medicine, 142 Shimokasuya, Isehara 160-8582, Japan

Received: 24 April 2013 Accepted: 8 May 2014

Published: 17 May 2014

\section{References}

1. Stahl NI, Klippel JH, Decker JL: A cutaneous lesion associated with myositis. Ann Intern Med 1979, 91(4):577-579.

2. Bohan A, Peter JB: Polymyositis and dermatomyositis (first of two parts). N Engl J Med 1975, 292(7):344-347.

3. Tanimoto K, Nakano K, Kano S, Mori S, Ueki H, Nishitani H, Sato T, Kiuchi T, Ohashi Y: Classification criteria for polymyositis and dermatomyositis. J Rheumatol Apr 1995, 22(4):668-674.

4. Mitra D, Lovell CL, Macleod TI, Tan RS, Maddison PJ: Clinical and histological features of 'mechanic's hands' in a patient with antibodies to Jo-1-a case report. Clin Exp Dermatol 1994, 19(2):146-148.

5. Garcia-Patos V, Bartralot R, Fonollosa V, Arnal C, Boronat M, Gelpí C, Rodríguez JL, Castells A: Childhood sclerodermatomyositis: report of a case with the anti-PM/Scl antibody and mechanic's hands. Br J Dermatol 1996, 135(4):613-616.

6. Watanabe C, Okubo Y, Ohi T, Koga M, Abe H, Tawara K, Tsuboi N, Hayashi T: A case of dermatomyositis associated with mechanic's hand. J Dermatol Nov 2000, 27(11):711-716.

7. Mittal R, Sharma VK, Prasad HR, Singh MK: Mechanic's hand: a clinical diagnostic aid in dermatomyositis. Acta Derm Venereol 2001, 81(1):65-66.

8. Taggart AJ, Finch MB, Courtney PA, Gormley GJ: Anti Jo-1 myositis. 'Mechanic's hands' and interstitial lung disease. Ulster Med J 2002, 71(1):68-71.

9. Shibuya H, Arakawa S, Kai Y, Shibuya H, Arakawa S, Kai Y, Hatano Y, Okamoto O, Takayasu S, Fujiwara S: Three cases of 'mechanic's hands' associated with interstitial pneumonia: possible involvement with foot lesions. J Dermatol Dec 2003, 30(12):892-897.

10. Torok L, Danko K, Cserni G, Szucs G: PM-SCL autoantibody positive scleroderma with polymyositis (mechanic's hand: clinical aid in the diagnosis). J Eur Acad Dermatol Venereol 2004, 18(3):356-359.

11. Hara H, Inoue Y, Sato T: Clinical and pathological findings of patients with interstitial lung disease associated with antisynthetase. Nihon Kokyuki Gakkai Zasshi 2005, 43(11):652-663.

12. White JM, Salisbury JR, Creamer D: Eczematous changes on the handsquiz case. Diagnosis: "Mechanic's hands" as part of the antisynthetase syndrome. Arch Dermatol 2005, 141(6):779-784.

13. Asanuma $Y$, Koichihara R, Koyama S, Asanuma Y, Koichihara R, Koyama S, Kawabata Y, Kobayashi S, Mimori T, Moriguchi M: Antisynthetase syndrome associated with sarcoidosis. Intern Med 2006, 45(18):1065-1068.

14. Plastiras SC, Soliotis FC, Vlachoyiannopoulos P, Tzelepis GE: Interstitial lung disease in a patient with antisynthetase syndrome and no myositis. Clin Rheumatol 2007, 26(1):108-111.

15. Bachmeyer C, Tillie-Leblond I, Lacert A, Cadranel J, Aractingi S: "Mechanic's hands": a misleading cutaneous sign of the antisynthetase syndrome. Br J Dermatol 2007, 156(1):192-194.

16. Yang CJ, Sheu CC, Ou TT, Hwang JJ, Huang MS: Combined lung fibrosis and 'mechanic's hand': a clinical diagnostic clue to amyopathic antisynthetase syndrome. Respirology 2008, 13(4):611-614.

17. Mii S, Kobayashi R, Nakano T, Mii S, Kobayashi R, Nakano T, Harada H, Okada M, Matsui Y, Fujii T, Eto H: A histopathologic study of mechanic's hands associated with dermatomyositis: a report of five cases. Int J Dermatol 2009, 48(11):1177-1182.

18. Shimizu K, Tai H, Kuwano K: A case of cellular NSIP with anti-OJ (anti-isoleucyl tRNA synthetase) antibodies. Nihon Kokyuki Gakkai Zasshi 2010, 48(1):45-48.
19. Bygum A, Velander MJ, Knudsen JB: Mechanic's hands imitating hand eczema. Dermatitis 2010, 21(6):334-335.

20. Seitz CS, Trautmann A: Differential diagnosis of mechanic's hands: think of Jo-1 syndrome. Contact Dermatitis 2010, 63(3):168-169.

21. Wendling D, Prati C: Mechanic's hands. Joint Bone Spine 2011, 78(4):419.

22. Patel HC, Lauder NN: The antisynthetase syndrome. Am J Med 2011, 124(9):e3-e4.

23. Christopher-Stine L, Robinson DR, Wu CC, Mark EJ: Case records of the Massachusetts General Hospital. Case 37-2012. A 21-year-old man with fevers, arthralgias, and pulmonary infiltrates. N Engl J Med 2012, 367(22):2134-2146.

24. Riveiro-Barciela M, Labirua-lturburu A, Selva-O'Callaghan A: A 79-year-old man with dyspnea, dysphagia, and weakness. Chest 2012, 142(1):252-255.

25. Sato Y, Teraki Y, Izaki S, Amano K: Clinical characterization of dermatomyositis associated with mechanic's hands. J Dermatol Dec 2012, 39(12):1093-1095.

26. Fischer A, Swigris JJ, du Bois RM, Lynch DA, Downey GP, Cosgrove GP, Frankel SK, Fernandez-Perez ER, Gillis JZ, Brown KK: Anti-synthetase syndrome in ANA and anti-Jo-1 negative patients presenting with idiopathic interstitial pneumonia. Respir Med 2009, 103(11):1719-1724.

27. Love LA, Leff RL, Fraser DD, Targoff IN, Dalakas M, Plotz PH, Miller FW: A new approach to the classification of idiopathic inflammatory myopathy: myositis-specific autoantibodies define useful homogeneous patient groups. Medicine (Baltimore) 1991, 70(6):360-374.

28. Watanabe K, Handa T, Tanizawa K, Hosono Y, Taguchi Y, Noma S, Kobashi Y, Kubo T, Aihara K, Chin K, Nagai S, Mimori T, Mishima M: Detection of antisynthetase syndrome in patients with idiopathic interstitial pneumonias. Respir Med 2011, 105(8):1238-1247.

doi:10.1186/1756-0500-7-303

Cite this article as: Sohara et al:: Mechanic's hands revisited: is this sign still useful for diagnosis in patients with lung involvement of collagen vascular diseases? BMC Research Notes 2014 7:303.

\section{Submit your next manuscript to BioMed Central and take full advantage of:}

- Convenient online submission

- Thorough peer review

- No space constraints or color figure charges

- Immediate publication on acceptance

- Inclusion in PubMed, CAS, Scopus and Google Scholar

- Research which is freely available for redistribution 